ENCYCLOPEDDIE Encyclopédie berbère

BERBERE

$31 \mid 2010$

31 | Matmora - Mezrag

\title{
Meules et molettes (Sahara)
}

M. Gast

\section{OpenEdition}

Journals

Édition électronique

URL : http://journals.openedition.org/encyclopedieberbere/586

DOI : $10.4000 /$ encyclopedieberbere.586

ISSN : 2262-7197

\section{Éditeur}

Peeters Publishers

\section{Édition imprimée}

Date de publication : 30 décembre 2010

Pagination : 4954-4959

ISBN : 978-90-429-2368-3

ISSN : 1015-7344

\section{Référence électronique}

M. Gast, « Meules et molettes (Sahara) », Encyclopédie berbère [En ligne], 31 | 2010, document M101b, mis en ligne le 08 octobre 2020, consulté le 13 octobre 2020. URL : http://journals.openedition.org/ encyclopedieberbere/586 ; DOI : https://doi.org/10.4000/encyclopedieberbere.586

Ce document a été généré automatiquement le 13 octobre 2020

(C) Tous droits réservés 


\title{
Meules et molettes (Sahara)
}

\author{
M. Gast
}

\section{Meule}

Les surfaces à broyer que l'on trouve au Sahara sont de deux sortes :

- Celles en place sur des blocs de granit ou de grès en plan horizontal.

- Celles sur des pierres mobiles plus ou moins lourdes, brutes de taille ou aménagées. Dans cette dernière catégorie existent des pièces anciennes façonnées par bouchardage sur les deux faces, légères et parfois décorées de sculptures.

2 On exclura toutes les traces d'usure par frottement en position verticale ou oblique sur des parois rocheuses, quelles que soient leurs dimensions.

3 L'objet transportable appelé plus communément "meule dormante " (parce que passive au sol), est une pierre plate dont la surface active devient légèrement concave à l'usage, et sur laquelle on frotte en percussion posée, oblique et diffuse, avec une ou deux mains, une autre pierre oblongue appelée "molette", pour broyer, moudre un matériau végétal (grain, écorce, feuilles, etc.), animal (viande séchée, os...) ou minéral (sel, natron, colorants minéraux...).

Comme les Touaregs, on distinguera deux sortes de matériel :

5 1. Les objets mobiles, de forme ovale, de profil bas ou galbé, de surface active concave d'environ 20 à $50 \mathrm{~cm}$ de longueur, larges de 20 à $30 \mathrm{~cm}$ et d'une profondeur variant de 0 à $6 \mathrm{~cm}$ environ par rapport au bord périphérique sont appelés édégé (auge) en tamâhaq (variante de l'Ahaggar).

6 Ces sortes de "plats » sont finement bouchardés sur leur surface extérieure réalisant une forme légère "en écaille », en général harmonieuse et régulière, d'une épaisseur moyenne de 2 à $4 \mathrm{~cm}$ environ. Ces "plats ", " auges ", " augets " sont parfois sculptés de décorations sur les bords (feston, reliefs divers) qui donnent à ces objets une valeur esthétique indéniable que certains auteurs ont rapprochée de celles des rondes-bosses.

7 2. Les meules dites "dormantes", fabriquées par les populations actuelles et que les Touaregs appellent tehunt $n$ azî̀ ou tehunt ta $n$ azîd (« pierre $^{1}$ de mouture "; meule de 
pierre, différenciée du moulin rotatif appelé tasyart) ou tašeffart. Ces objets ont des formes quelconques (rectangulaires, carrées, circulaires) brutes de retouche ou sont grossièrement épannelés, toujours très épais, de 7 à $20 \mathrm{~cm}$ ou même davantage. Choisies le plus souvent en grès, offrant une surface naturelle plane, ces meules acquièrent à l'usage une ensellure de plus en plus prononcée due à la friction de la molette et au bouchardage ravivant le mordant de la surface active. Dans le cas de broyage de céréales, la surface utile peut avoir entre 15 et $25 \mathrm{~cm}$ de long et d'environ 9 à $15 \mathrm{~cm}$ de large, selon les dimensions de la molette utilisée. Ces meules bien que transportables, sont en général lourdes, encombrantes et ne font pas l'objet d'une appropriation particulière ; les nomades les laissent sur les lieux de leurs campements et les retrouvent à chacun de leurs retours. Elles peuvent aussi servir aux voyageurs de passage. Quant aux sédentaires, ils les laissent dans les huttes, les maisons et les espaces de leurs activités en les retournant (comme les nomades), la surface active contre le sol, pour la protéger des souillures et, selon eux, des génies.

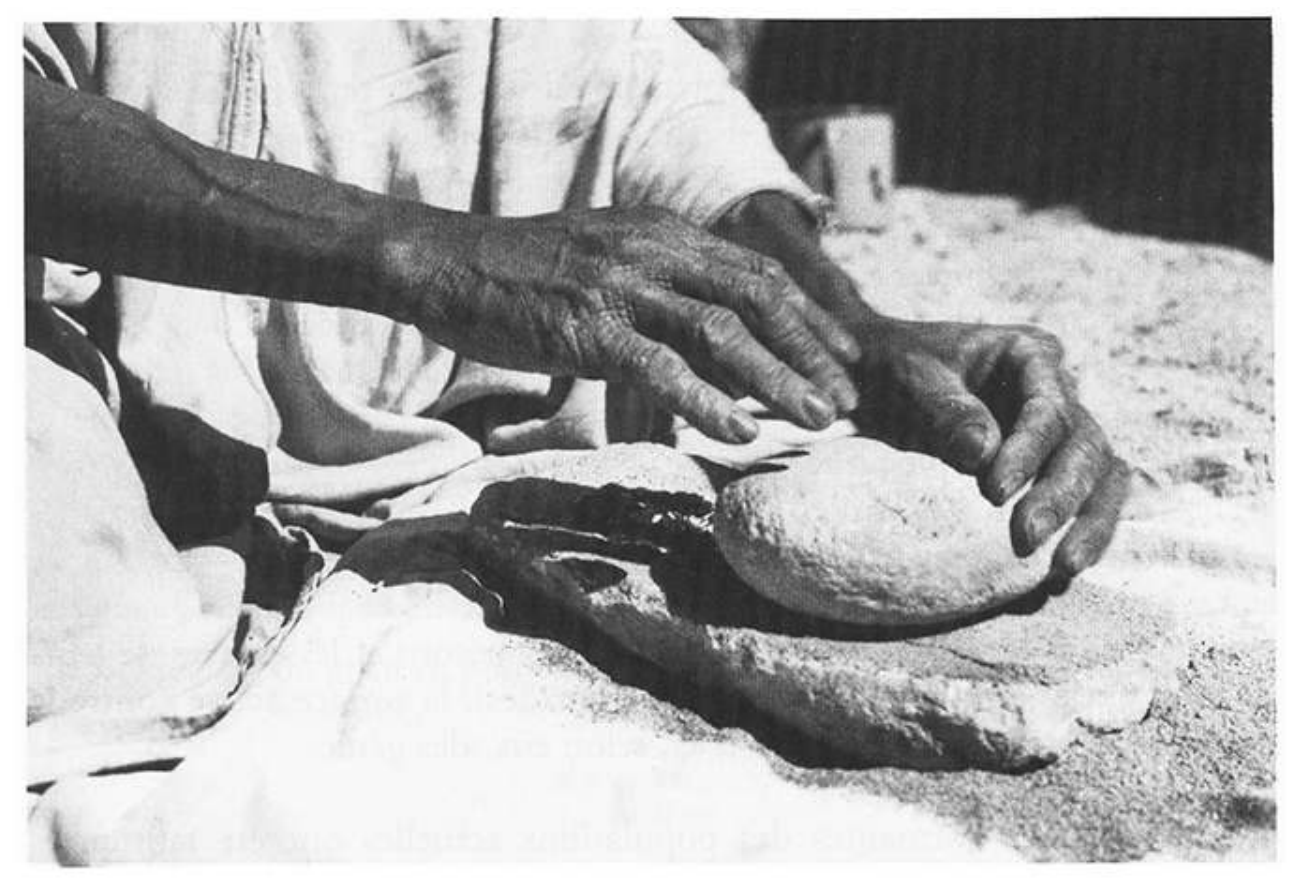

1. PRÉPARATION D'UNe MEULE ET de SA MOLETTE PAR BOUCHARDAge, À L'AIDE D'UN PERCUTEUR DE QUARTZITE (MAHDI EL-KHERREZ À IDÉLÈS, AHAggAR). 


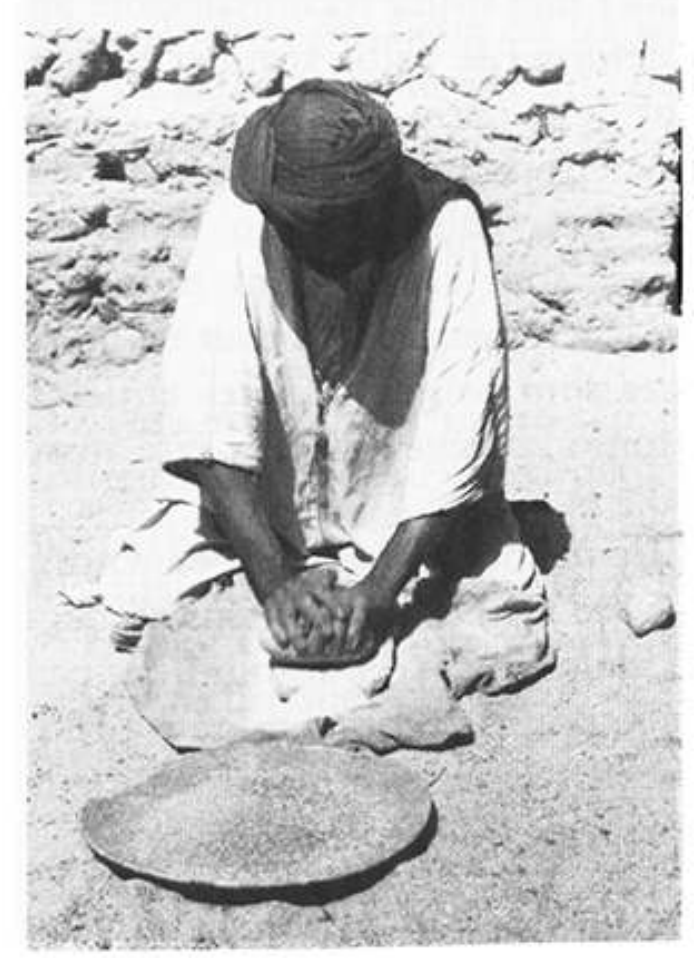

2. MEULAgE du gRAIN EN POSITION ASSISE.

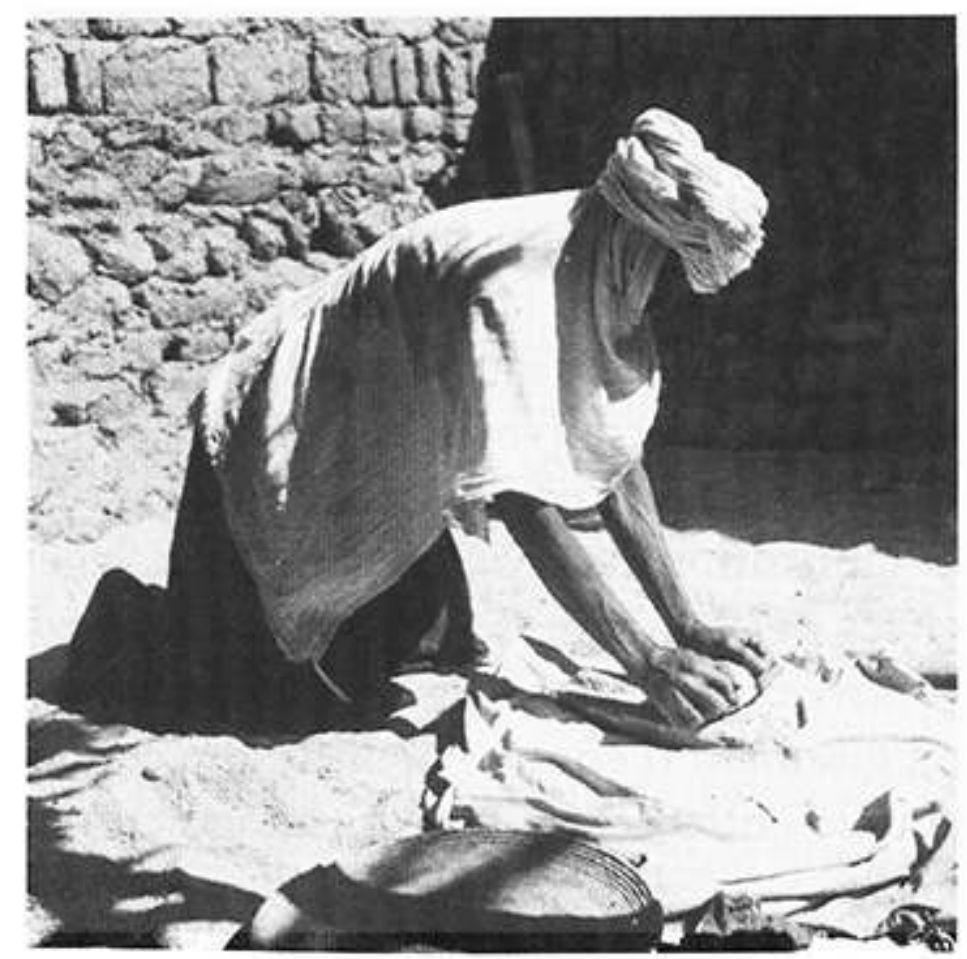

3. PLAT RÉEMPLOYÉ COMME MEULE, PERForé PAR USURE EN SON MILIEU.

8 Si les meules dormantes des populations actuelles ont été fabriquées pour broyer des graines cultivées, des végétaux ou quelquefois des os ou des minéraux, aucune certitude n'a jamais été établie sur l'âge véritable, l'emploi et les raisons d'être des 
objets les plus anciens. Cependant, ces derniers fréquents en particulier sur les grands ergs (Admer, Issaouane, Tihigaline, Ténéré...), en surface ou légèrement enfouis dans le sable, ont été souvent réutilisés par les populations actuelles pour broyer des graines cultivées (blé, orge ou, exceptionnellement, sorgho et mil habituellement traités au mortier de bois). Ces usages provoquent alors une usure reconnaissable au recreusement de l'ensellure primitive qui déforme le galbe régulier de ces surfaces en provoquant parfois le percement de la paroi.

L'hypothèse d'un usage de ces "plats » anciens pour broyer les graines de cueillette consommées encore aujourd'hui dans l'alimentation humaine n'est pas recevable. Car ces graines sauvages sont simplement mondées entre les deux paumes de mains, ou traitées légèrement au mortier de bois, étant donné leur faible granulométrie. Elles sont toujours cuites entières sans être broyées, sauf quelquefois pour le fonio sauvage qui peut être aussi broyé à la pierre (voir Gast 1995).

Par ailleurs, l'hypothèse d'une « agriculture néolithique » ayant engendré la fabrication de ces objets minces, légers et de formes visiblement recherchées par leur finesse et parfois décorés de sculptures n'est absolument pas prouvée. Aucune graine d'espèce cultivée n'a, pour le moment, été trouvée en gisement d'époque néolithique et aucune corrélation n'a pu être dûment établie entre ces plats et un usage en rapport avec une agriculture.

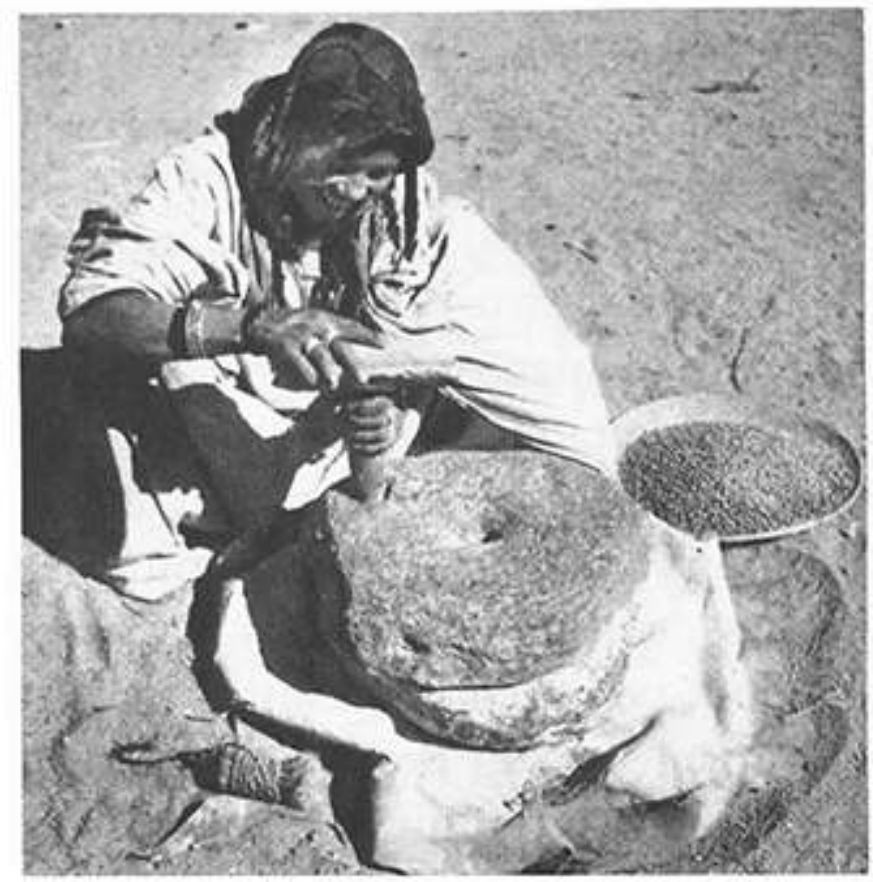

3. meulage du grain en POSition Agenouillée. 


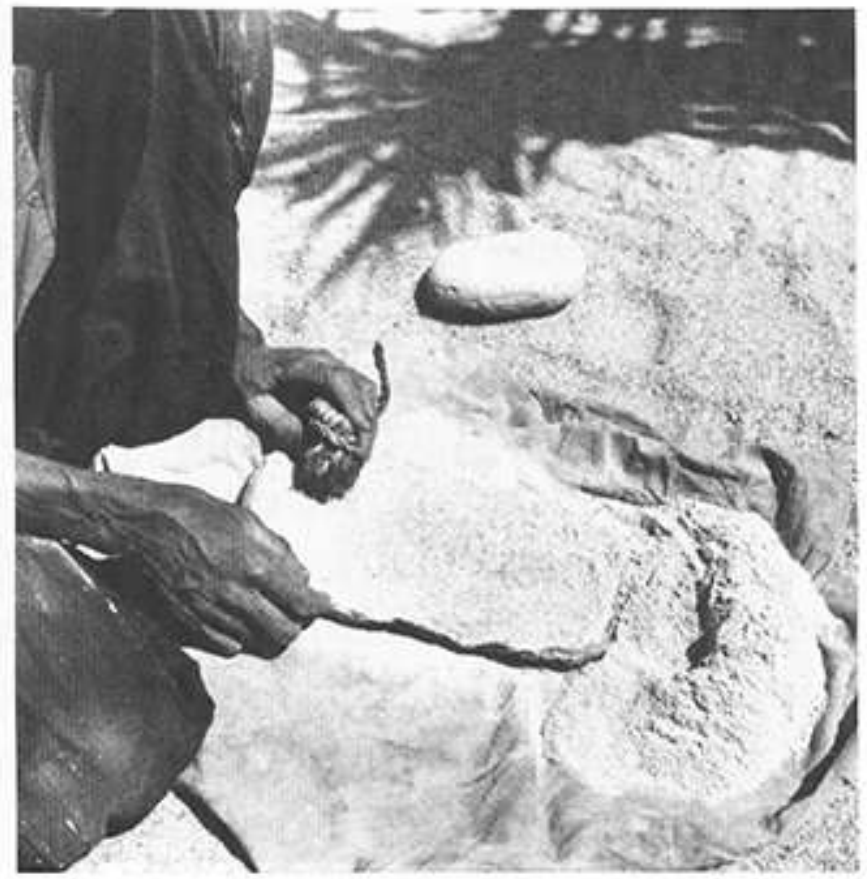

4. SURFACES DE BROYAgE SUR gRANIT EN BOULE À AMEKNI (AHAggAR). DIMENSIONS MOYENNES : L. 25 À 30 CM, L. 10 À 15 CM, P. 7 À 12 CM.

Enfin, toutes les surfaces de broyage notamment en place sur des reliefs de granit ou de grès ne peuvent être dénommées "meules». Bon nombre de ces surfaces d'usure se trouvent soit sur des dalles horizontales portant des gravures rupestres (dessins, représentations animales notamment), soit formant des cavités oblongues étroites et profondes, très proches les unes des autres, sur un même site. La raison d'être de ces frottis et creusements anciens, ainsi que leur fonction originelle, n'ont pas été élucidées. Cependant, il existe des lieux où les voyageurs viennent encore aujourd'hui faire des vœux en frottant sur une paroi, un rocher, reconnus bénéfiques, pour obtenir une poussière dont ils s'enduisent le visage ou pour faire des talismans protecteurs.

\section{Molette}

12 C'est un bloc de pierre qui peut être de forme oblongue, cylindrique, sphérique, en fuseau ou en petit "pavé ", préhensible à une ou deux mains, qui sert à écraser des substances végétales ou minérales sur une meule, par percussion posée, oblique et diffuse.

Les données ethnologiques fournissent quelques points de repère de références techniques qui éclairent la conception et le fonctionnement de ces outils.

Les dimensions de la molette sont établies en fonction de celles de la surface de la meule et de la nature du produit à broyer. L'homme qui prépare une molette, choisit soit un galet, soit une pierre d'une dimension adéquate en l'aménageant par bouchardage. La nature de la roche de la molette est en général différente de celle de la meule; elle doit être plus dure et plus résistante que celle-ci. Si la meule est en grès, une molette en quartzite lui conviendra. 

elle perd ses caractères abrasifs sur la première face. La molette peut servir par ailleurs de percuteur dans toutes sortes d'opérations. On utilise alors, non pas la partie agissante dans le meulage, mais à tour de rôle les extrémités latérales pour enfoncer un piquet, écraser des noyaux de dattes, du sucre, du sel ou des épices, etc. Ce mouvement en percussion lancée perpendiculaire ou quelque fois diffuse, marque les extrémités de petits éclats caractéristiques, révélateurs des multiples fonctions dévolues aux molettes qui deviennent tour à tour pilon, percuteur, écrasoir, broyeur.

Cependant, la variété des volumes, des formes et du poids des molettes sahariennes trouvées en surface ou en gisement, conduit à penser à des spécialisations artisanales autres que celle de la mouture de grains ou de végétaux. La finesse du polissage de certaines pièces, leur petitesse, pourraient les désigner comme instrument à corroyer le cuir, à réduire en poudre fine certains colorants par exemple.

Si l'on a trouvé en couches archéologiques, dans nombre de gisements, des outils que l'on peut qualifier de molettes et quelques mortiers, les meules y sont plutôt rares.

Une étude extensive, globale, des fonctions supposées des pièces collectionnées en surface sur les vastes étendues sahariennes, paraît vaine et prématurée; car il semble essentiel de connaître l'environnement écologique et l'échelle du temps dans lesquels l'on peut placer chaque objet, pour approcher le potentiel de leurs usages et de leurs fonctions.

\section{Techniques d'utilisation}

Sur une meule dormante, réservée à la monture de grains et de matières sèches, le travail s'effectue selon deux positions du corps :

- à genoux, fesses posées sur les talons, les bras en extension avant, les deux mains posées sur la pierre.;

- à genoux, le corps penché vers l'avant, les jambes en extension régulant le mouvement de va et vient des bras et du corps qui offrent alors le maximum de pression sur la pierre.

Cette technique de broyage détermine sur les molettes servant à meuler du blé, de l'orge ou du sorgho, une usure particulière : léger front à l'avant de l'outil, usure en pente douce vers l'arrière et qui tend à amincir l'épaisseur de la molette. D'autres déformations apparaissent aussi selon le sens de la poussée déviant un peu à droite ou à gauche et qui se remarquent aussi sur la meule.

Le rendement de ce travail dépend de la force de l'ouvrier, de sa position, et des caractéristiques techniques du produit à moudre (blé dur, blé tendre, orge, riz, sorgho, etc.). Un homme de force normale peut moudre de 3 à $4 \mathrm{~kg}$ de blé en une heure sur une meule qui offre une surface utile de 15 à $25 \mathrm{~cm}$ de long sur 10 à $15 \mathrm{~cm}$ de large environ. Mais les temps peuvent varier énormément selon la granulométrie de la mouture recherchée. Une mouture fine exige plusieurs passages sur la poignée de grain disposée au départ de la friction. Les molettes comme les meules peuvent subir des ravivages à l'aide de percuteurs réservés à cet effet; les blocs de quartz de la taille d'un poing sont particulièrement appréciés pour ce travail. Mais les molettes subissent beaucoup moins fréquemment que les meules cette opération de ravivage.

Toutes les photos de la notice sont de M. Gast. 


\section{BIBLIOGRAPHIE}

CAMPS-FABRER H., Matière et art mobilier dans la préhistoire nord-africaine et saharienne, (Mémoire du CRAPE, V, Alger), Paris, AMG, 1966, p. 285-286.

GAST M., « Des graines nourricières qu'on ne broie pas : les confusions de « l'agriculture saharienne » » in L'Homme méditerranéen. Mélanges offerts à Gabriel Camps. Publications de l'Université de Provence, Aix-en-Provence, 1995, p. 249-257.

ROUBET C., Essai de classification du matériel de broyage, Actes du VIII congrès des sciences préhistoriques et protohistoriques, Beograd, 1973, p. 413-417.

ROSET J. P., « Une meule néolithique ornée du Ténéré (Sahara nigérien) », Archéologia, 58, 1973, p. 66-68.

RouX V., Le matériel de broyage, Etude ethnoarchéologique à Tichitt (R.I Mauritanie), Mémoire ${ }^{\circ}$ 58, Ed. Recherche sur les civilisations, Paris ADPF, 1985, p. 112.

SAVARY J. P., « Note sur une meule néolithique ornementée provenant de Tihigaline (Sahara oriental) », Bull. de la Soc. Préhist. Franç., 1963, p. 361.

\section{NOTES}

1. Le terme tehunt est pan-berbère et se retrouve dans les autres dialectes berbères sous les formes tawunt, tagunt, taggunt, où il signifie: « rocher massif de forme tabulaire, pierre lourde servant de surface de travail, enclume...»

\section{INDEX}

Mots-clés : Agriculture, Alimentation, Ethnologie, Ethnographie, Sahara, Technologie, Touareg 